\title{
The $\eta_{c} \gamma \gamma^{*}$ transition form factor in the decay $\eta_{c} \rightarrow \gamma \ell^{+} \ell^{-}$and in the crossed channels $\gamma e^{-} \rightarrow \eta_{c} e^{-}$and $e^{+} e^{-} \rightarrow \eta_{c} \gamma$.
}

\author{
Elvio Di Salvo \\ Dipartimento di Fisica dell' Università di Genova and I. N. F. N. - Sez. Genova, Via \\ Dodecaneso 33, 16146 Genova, Italy \\ Michail P. Rekalo * \\ Middle East Technical University, Physics Department, Ankara 06531, Turkey \\ Egle Tomasi-Gustafsson \\ DAPNIA/SPhN, CEA/Saclay, 91191 Gif-sur-Yvette Cedex, France
}

(November 14, 2018)

\begin{abstract}
The Dalitz distribution and the effective mass spectrum of the lepton pair produced in the decay $\eta_{c} \rightarrow \gamma \ell^{+} \ell^{-}$, as well as the differential cross sections for the crossed channels $\gamma e^{-} \rightarrow \eta_{c} e^{-}$and $e^{+} e^{-} \rightarrow \eta_{c} \gamma$, are calculated in terms of the sole electromagnetic transition form factor for the vertex $\eta_{c} \rightarrow \gamma \gamma^{*}$, where $\gamma^{*}$ is a virtual photon with a space-like (for $\gamma e^{-} \rightarrow \eta_{c} e^{-}$) or a time-like (for $e^{+} e^{-} \rightarrow \eta_{c} \gamma$ and $\left.\eta_{c} \rightarrow \gamma \ell^{+} \ell^{-}\right)$four-momentum. Explicit parametrisations of the transition form factor are found in two different approaches, namely in Vector Dominance Model and in a QCD-inspired consideration on the basis of the process $c \bar{c} \rightarrow \gamma \ell^{+} \ell^{-}$, with zero relative three-momentum of the annihilating quarks.
\end{abstract}

\section{INTRODUCTION}

The radiative decays of mesons and baryons are an interesting source of important information for hadron spectroscopy and hadron electrodynamics [1].2]. For example, it is worth recalling the role of such decays in determining the charmonium spectrum [2]. Among radiative decays, especially intriguing are those where lepton pairs are produced. A classical example is the process $\pi^{0} \rightarrow \gamma e^{+} e^{-}$[3], which is sensitive to the radius of the corresponding form factor. Such a decay is typical of any neutral pseudoscalar meson $P\left(=\eta, \eta^{\prime}, \eta_{c}\right.$, etc.):

$$
P \rightarrow \gamma \ell^{+} \ell^{-}, \quad \ell=e, \mu .
$$

\footnotetext{
*Permanent address: National Science Center KFTI, 310108 Kharkov, Ukraine
} 
The corresponding transition electromagnetic form factors of $P \rightarrow \gamma \gamma^{*}$-decay are complex functions of $t$, the four-momentum transfer squared, in the time-like region $4 m_{\ell}^{2}<t \leq m^{2}$, where $m\left(m_{\ell}\right)$ is the mass of $P(\ell)$. In particular the decay $\eta_{c} \rightarrow \gamma \ell^{+} \ell^{-}$offers the possibility of studying the transition form factor in a relatively large region of time-like momentum transfer,

$$
4 m_{\ell}^{2} \leq t \leq 9 \mathrm{GeV}^{2} .
$$

A strong theoretical interest in the transition form factors of pseudoscalar mesons was stimulated by the experimental results of the CLEO collaboration [4,5], which measured these form factors at space-like momentum transfers, using the well known $2 \gamma$-exchange mechanism in the reaction $e^{+} e^{-} \rightarrow e^{+} e^{-} P$ (Fig. 1). If one lepton, say the positron, is not detected, we can study the process of photoproduction of pseudoscalar mesons on electrons, $\gamma e^{-} \rightarrow P e^{-}$, in an unusual kinematics of colliding particles, $\gamma$ and $e^{-}$. In the case of $\pi^{0}$ production the corresponding form factor was measured up to $t=-8 \mathrm{GeV}^{2}$, where, in principle, perturbative QCD applies [6 9]. Recently also the $\eta_{c} \gamma$ transition form factor has been determined [10] for two different values of $t$ in the spacelike region.

A special interest has the decay $\eta_{c} \rightarrow \gamma \ell^{+} \ell^{-}$, as a generalisation of the decay $\eta_{c} \rightarrow \gamma \gamma$, whose branching ratio is known [11,10] (see also predictions by dispersive methods [12 [16]). The availability of a form factor instead of a single constant, as in the case of $\eta_{c} \rightarrow 2 \gamma$ decay, allows a better insight of the structure of the $\eta_{c}$-meson and of perturbative QCD. It is also interesting to look at the predictions of the "old" approach of Vector Dominance Model (VDM) in the unusual region of charmed particle decays [17].

This paper is devoted to a global approach to the study of all possible crossed channels, i. e.,

$$
\eta_{c} \rightarrow \gamma \ell^{+} \ell^{-}, \quad \gamma e^{-} \rightarrow \eta_{c} e^{-}, \quad e^{+} e^{-} \rightarrow \eta_{c} \gamma,
$$

whose characteristics are driven by a single transition form factor. The numerical estimates are performed both within VDM and QCD, considering, in the latter case, the process $c \bar{c} \rightarrow \gamma \ell^{+} \ell^{-}$as the basic mechanism generating the $\eta_{c} \rightarrow \gamma \gamma *$ transition form factor.

Section II is dedicated to the Dalitz plot distribution of the decay $\eta_{c} \rightarrow \gamma \ell^{+} \ell^{-}$. In sect. III we propose two models for calculating the transition form factor $\eta_{c} \rightarrow \gamma \gamma *$, one based on VDM and the other on QCD. In sect. IV we study the reaction $e^{+} e^{-} \rightarrow \eta_{c} \gamma$. Sect. V is dedicated to the process $\gamma e^{-} \rightarrow \eta_{c} e^{-}$. Lastly in sect. VI we draw some conclusions.

\section{THE DECAY $\eta_{c} \rightarrow \gamma \ell^{+} \ell^{-}$.}

We start by considering the more general case of the decay of a meson $M$ into a real $\gamma$ and a lepton pair. The differential decay width reads

$$
d \Gamma=(2 \pi)^{4} \frac{\overline{|\mathcal{M}|^{2}}}{2 m} \delta^{4}\left(p-q-k_{1}-k_{2}\right) \frac{d^{3} q}{(2 \pi)^{3} 2 \omega} \frac{d^{3} k_{1}}{(2 \pi)^{3} 2 E_{1}} \frac{d^{3} k_{2}}{(2 \pi)^{3} 2 E_{2}},
$$

where $\omega, E_{1}$ and $E_{2}$ are the energies of the final $\gamma, e^{+}$and $e^{-}$respectively.

The notation of the four-momenta is shown in Fig. 2; the line above the modulus squared of the matrix element $|\mathcal{M}|^{2}$ denotes the sum over the polarisations of all the particles produced in the decay. 
The standard form of the matrix element $\mathcal{M}$ for the $M \rightarrow \gamma \ell^{+} \ell^{-}$decay in one-photon approximation is

$$
\mathcal{M}=\frac{e^{2}}{t} \ell_{\mu} \mathcal{J}^{\mu}
$$

where $t=\left(k_{1}+k_{2}\right)^{2}$ is the effective mass squared of the leptonic pair, $\ell_{\mu}=\bar{u}\left(k_{2}\right) \gamma_{\mu} u\left(-k_{1}\right)$ and $\mathcal{J}_{\mu}$ is the electromagnetic current in the decay $M \rightarrow \gamma \gamma^{*}$ 円.

According to the usual notation we obtain

$$
\overline{|\mathcal{M}|^{2}}=\frac{e^{4}}{t^{2}} L_{\mu \nu} W^{\mu \nu}
$$

where

$$
L_{\mu \nu}=\overline{\ell_{\mu} \ell_{\nu}^{*}}, \quad W^{\mu \nu}=\overline{\mathcal{J}^{\mu} \mathcal{J}^{\nu *}}
$$

Substituting (2) into (1), and integrating over all variables except $E_{1,2}$, we get the two-lepton Dalitz distribution:

$$
d \Gamma=\frac{\alpha^{2}}{4 \pi} \frac{L_{\mu \nu} W^{\mu \nu}}{t^{2} m} d E_{1} d E_{2},
$$

where $\alpha=e^{2} / 4 \pi$. However for our aims it is more convenient to express $E_{1}$ and $E_{2}$ in terms of the dimensionless variables $x=t / m^{2}$ and $y=2\left(E_{1}-E_{2}\right) / m$ [18, which are defined in the ranges $\delta \leq x \leq 1$ and $-y_{0} \leq y \leq y_{0}, y_{0}=(1-x) \sqrt{1-\delta / x}$ and $\delta=4 m_{\ell}^{2} / m^{2}$. Furthermore we take into account, wherever possible, symmetry properties:

- Gauge invariance $-k \cdot \ell=k \cdot \mathcal{J}=0$ - implies that the product $L_{\mu \nu} W^{\mu \nu}$ can be rewritten in terms of the space components of the two tensors above, i. e.,

$$
L_{\mu \nu} W^{\mu \nu}=\left(L_{x x}+L_{y y}\right) W_{x x}+\frac{t^{2}}{k_{0}^{4}} L_{z z} W_{z z}
$$

where $k_{o}$ denotes the energy of the $\gamma^{*}$. Here we have adopted a reference frame in which the meson $M$ is at rest and the $z$-axis is parallel to the momentum $\vec{k}$ of the virtual photon.

- Parity invariance allows to write

$$
W_{i j}=\left(\delta_{i j}-\hat{k_{i}} \hat{k_{j}}\right) W_{1}(t)+\hat{k_{i}} \hat{k_{j}} W_{2}(t), \quad \hat{\vec{k}}=\vec{k} /|\vec{k}|,
$$

where $W_{1(2)}(t)$ is the real structure function $(\mathrm{SF})$ which describes the production of $\gamma^{*}$ with transverse (longitudinal) polarisation.

Taking into account all that, calculations yield

$$
d \Gamma=\frac{\alpha^{2}}{16 \pi} W_{1}(t) \frac{d x d y}{x m}\left[1+\frac{4 m_{\ell}^{2}}{t}+R_{L}(t) \frac{t}{k_{0}^{2}}+y^{2} \frac{m^{2}}{4 \vec{k}^{2}}\left(1-R_{L}(t) \frac{t}{k_{0}^{2}}\right)\right],
$$

\footnotetext{
${ }^{1}$ In the decay considered here, $\mathcal{J}_{\mu}$ is proportional to the electric charge $e$.
} 


$$
R_{L}(t)=W_{2}(t) / W_{1}(t), \quad k_{0}=\frac{m^{2}+t}{2 m}, \quad \vec{k}^{2}=\frac{\left(m^{2}-t\right)^{2}}{4 m^{2}} .
$$

One can see that the differential width, which characterises the Dalitz distribution for the decay $M \rightarrow \gamma \ell^{+} \ell^{-}$, is symmetric with respect to the exchange $E_{1} \leftrightarrow E_{2}$, in agreement with $C$-invariance of the electromagnetic interaction.

The specific dependence of $d^{2} \Gamma / d x d y$ on the variable $y$,

$$
d^{2} \Gamma / d x d y=a(x)+y^{2} b(x),
$$

is a direct consequence of one-photon mechanism. It has the same origin as the $\cot ^{2} \frac{\theta_{e}}{2}$ dependence of the differential cross section in electron-hadron scattering (where $\theta_{e}$ is the electron scattering angle in the laboratory system) and as the $\cos ^{2} \theta$-dependence of the differential cross section for the inclusive process $e^{+} e^{-} \rightarrow h X$, where $h$ denotes the final detected hadron and $\theta$ is the angle between the three-momenta of the hadron and of the electron in the overall CMS.

One can see that the value $W_{1}(0)$ determines the probability of the radiative decay width $\Gamma_{0} \equiv \Gamma(M \rightarrow 2 \gamma)$, i.e.,

$$
\Gamma_{0}=\frac{1}{2}(2 \pi)^{4} e^{2} \frac{W_{x x}+W_{y y}}{2 m} \int \delta^{4}(p-q-k) \frac{d^{3} k}{(2 \pi)^{3} 2 \omega_{1}} \frac{d^{3} q}{(2 \pi)^{3} 2 \omega_{2}}=\frac{\alpha}{4 m} W_{1}(0) .
$$

This allows to rewrite the Dalitz distribution in the following form:

$$
\frac{1}{\Gamma_{0}} \frac{d^{2} \Gamma}{d x d y}\left(M \rightarrow \gamma \ell^{+} \ell^{-}\right)=\frac{\alpha}{4 \pi} R_{T}(t) \frac{1}{x}\left[1+\frac{4 m_{\ell}^{2}}{t}+R_{L}(t) \frac{t}{k_{0}^{2}}+\frac{y^{2}}{4 \overrightarrow{k^{2}}} m^{2}\left(1-R_{L}(t) \frac{t}{k_{0}^{2}}\right)\right],
$$

where $R_{T}(t)=W_{1}(t) / W_{1}(0)$ and $R_{L}(t)$ is defined in Eq. (5)).

Therefore the study of the energy distribution of leptons in $M \rightarrow \gamma \ell^{+} \ell^{-}$in the Dalitz plane allows to determine $R_{L}(t)$ and $R_{T}(t)$, i. e., the two fundamental quantities of the decay considered, as functions of the effective mass of the $\ell^{+} \ell^{-}$-system.

The result of the integration in Eq. (6) over the variable $y$ determines the $\ell^{+} \ell^{-}$effective mass spectrum in the decay $M \rightarrow \gamma \ell^{+} \ell^{-}[19$ :

$$
\frac{1}{\Gamma_{0}} \frac{d \Gamma}{d x}\left(M \rightarrow \gamma \ell^{+} \ell^{-}\right)=\frac{2 \alpha}{3 \pi} R_{T}(t) \frac{(1-x)}{x}\left(1+2 \frac{m_{\ell}^{2}}{t}\right) \sqrt{1-\frac{4 m_{\ell}^{2}}{t}}\left[1+R_{L}(t) \frac{t}{2 k_{0}^{2}}\right] .
$$

Turning to the specific decay $\eta_{c} \rightarrow \gamma \gamma^{*}$, in this case (and more generally for any neutral pseudoscalar meson) the hadron electromagnetic current reads

$$
\mathcal{J}_{\mu}=\frac{F(t)}{m} \epsilon_{\mu \alpha \beta \gamma} k^{\alpha} e^{\beta} q^{\gamma}
$$

where $e_{\beta}$ is the polarisation four-vector of the real photon and $F(t)$ the electromagnetic form factor of the transition $\eta_{c} \rightarrow \gamma \gamma^{*}$, which has a nonzero imaginary part for $t \geq 4 m_{\pi}^{2}$. Note that we have defined the form factor differently from Lepage and Brodsky [20]. Inserting Eq. (8) into the definition of the tensor $W_{\mu \nu}$, Eq. (3), we obtain

$$
W_{1}(t)=|F(t)|^{2}(1-x)^{2} \frac{m^{2}}{4}, \quad W_{2}(t)=0
$$

i.e. $R_{L}(t)=0$.

There are three different processes for studying the $t$-dependence of $F$ : 
1. The photoproduction of $\eta_{c}$ in $\gamma e^{-} \rightarrow \eta_{c} e^{-}$at space-like momentum transfers;

2. The decay $\eta_{c} \rightarrow \gamma \ell^{+} \ell^{-}$, at time-like momentum transfers, $4 m_{\ell}^{2} \leq t \leq m^{2}$;

3. The reaction $e^{+} e^{-} \rightarrow \eta_{c} \gamma$, at time-like momentum transfers, $t \geq m^{2}$.

Therefore the simultaneous study of all these different processes can allow to determine the $t$-dependence of the electromagnetic form factor $F(t)$ for any value of $t$ (see Fig. 3 ). Moreover all the possible observables in the above mentioned processes depend only on $|F(t)|^{2}$. This means that the phase of $F(t)$ in the time-like region cannot be measured. But the determination of this phase as a function of $t$ is very important. For example, a dispersion relation for the form factor $F(t)$, considered as an analytical function of $t$, can be analysed only if both the modulus and the phase of $F(t)$ are known. The problem of measuring the phase of complex form factors of hadrons in the time-like region is not yet solved. This is a common problem of the electromagnetic form factor of the charged pion, of the neutral and charged kaons, etc..

The determination of the transition form factor $F(t)$ is necessary for calculating the following observables:

- The Dalitz-distribution in the variables $x$ and $y$;

- The spectrum of the effective masses in $\eta_{c} \rightarrow \gamma \ell^{+} \ell^{-}$;

- The coefficient of internal conversion, i.e. the ratio of the decay width of $\eta_{c} \rightarrow \gamma \ell^{+} \ell^{-}$ to the decay width of $\eta_{c} \rightarrow \gamma \gamma$ :

$$
I=\frac{\Gamma\left(\eta_{c} \rightarrow \gamma \ell^{+} \ell^{-}\right)}{\Gamma_{0}}=\frac{2 \alpha}{3 \pi} \int_{\delta}^{1} d x\left|\frac{F(t)}{F(0)}\right|^{2} \frac{(1-x)^{3}}{x}\left(1+2 \frac{m_{\ell}^{2}}{t}\right)\left(1-\frac{4 m_{\ell}^{2}}{t}\right)^{1 / 2} .
$$

This coefficient derives its main contribution from small values of $t$, therefore it is sensitive to the mass of the lepton, in particular it is different in the decays $\eta_{c} \rightarrow \gamma e^{+} e^{-}$and $\eta_{c} \rightarrow \gamma \mu^{+} \mu^{-}$.

\section{TWO MODELS FOR $F(t)$}

Now we consider the predictions of two models, the VDM and a pQCD-inspired nonrelativistic model.

\section{A. Vector Dominance Model}

In the framework of VDM (Fig. 4), the $t$-dependence of the electromagnetic form factor $F(t)$ can be written as

$$
F(t)=\sum_{V} \frac{g_{V}}{t-m_{V}^{2}}, \quad g_{V}=m_{V}^{2} f_{V} g\left(V \rightarrow \eta_{c} \gamma\right)
$$

having summed over the contributions of the vector mesons. $m_{V}$ is the vector meson mass, $f_{V}$ is the constant of the $\gamma^{*} \rightarrow V$-transition and $g\left(V \rightarrow \eta_{c} \gamma\right)$ is the constant in the vertex 
$V \rightarrow \eta_{c} \gamma$. If $m_{V} \geq m$, the decay $V \rightarrow \eta_{c} \gamma$ is possible and the corresponding branching ratio allows to determine the constant $g\left(V \rightarrow \eta_{c} \gamma\right)$, or, more exactly, as previously discussed, the modulus of this constant. Incidentally, notice that the constant $f_{V}$ determines the decay width of $V \rightarrow \ell^{+} \ell^{-}$(see Appendix).

The constants $g_{V}$ satisfy two important conditions:

$$
\begin{aligned}
-\sum_{V} \frac{g_{V}}{m_{V}^{2}} & =F(0), \\
\sum_{V} g_{V} & =0 .
\end{aligned}
$$

The first one is a relation between different $g_{V}$, through an evident normalisation of $F(t)$. The constant $F(0)$ can be determined from the decay width of $\eta_{c} \rightarrow 2 \gamma$, i.e., from

$$
\Gamma_{0}=\frac{\alpha}{4}|F(0)|^{2} m,
$$

which is experimentally known. Again, the value of the width $\Gamma_{0}$ does not fix the sign of $F(0)$, which introduces a two-fold ambiguity in relation (11).

Relation (12) results from a specific asymptotic behaviour of $F(t)$ : the structure of the current $\mathcal{J}_{\mu}$ shows that $F(t) \propto t^{-2}$ for large $|t|$. This differs from the standard $|t|^{-1}$ behaviour of the elastic electromagnetic form factors of mesons, however it agrees with the quark counting rule, and it results from the presence in Eq. (8) of an additional four-momentum, owing to the electromagnetic current of the decay considered.

The simplest model that can be suggested here for $F(t)$ must contain the contribution of two vector mesons. The problem is how to select the most important contributions.

Taking into account the $c \bar{c}$-nature of $\eta_{c}$, the $J / \psi$ seems to be a good candidate, as confirmed by the value of the branching ratio $\operatorname{Br}\left(J / \psi \rightarrow \eta_{c} \gamma\right)=(1.3 \pm 0.4) \%$. Similarly the branching ratio $\operatorname{Br}\left(\eta_{c} \rightarrow \rho \rho\right)=(2.6 \pm 0.9) \%$ exhibits the importance of the $\rho$-contribution to the form factor $F(t)$. Therefore in such a two-pole model we find the following two parametrisations:

$$
F(t)= \pm|F(0)| \frac{m_{\rho}^{2} m_{J / \psi}^{2}}{m_{J / \psi}^{2}-m_{\rho}^{2}}\left(\frac{1}{t-m_{\rho}^{2}}-\frac{1}{t-m_{J / \psi}^{2}}\right),
$$

which cannot be disentangled in the study of the above mentioned processes.

In order to avoid singularities at $t=m_{V}^{2}$, we have to substitute $m_{V}^{2} \rightarrow m_{V}^{2}-i m_{V} \Gamma_{V}$, where $\Gamma_{V}$ is the width of the $V$-meson $(V=\rho, J / \psi)$.

These parametrisations offer a numerical estimation of all the observables of the $\eta_{c}$ photoproduction on electrons, $\gamma e^{-} \rightarrow \eta_{c} e^{-}$, and of the decay $\eta_{c} \rightarrow \gamma \ell^{+} \ell^{-}$, where $t \leq m^{2}$. In both processes the $\rho$-contribution is particularly important at relatively small momentum transfers, $|t| \leq 1 \mathrm{GeV}^{2}$. The $J / \psi$-contribution plays an important role in the decay $\eta_{c} \rightarrow \gamma \ell^{+} \ell^{-}$, near the upper kinematical limit in the variable $t, t \rightarrow m^{2}$, where the $J / \psi$-pole is very close to the physical region.

On the other hand this simplified parametrisation is not convenient for the study of the annihilation process, $e^{+} e^{-} \rightarrow \eta_{c} \gamma$, to which the entire set of $\psi$-resonances in principle could contribute. In this case formula (14) has to be generalised, taking into account, at least,

- the contribution of additional $\psi$ resonances; 
- the widths of such resonances, as illustrated above.

In the framework of VDM it is in principle possible to write a five-pole representation for $F(t)$, with the contributions of $\rho, J / \psi, \psi(3.77), \psi(4.04), \psi(4.41)$. The constants $g_{V}$ of $\rho, J / \psi$ and $\psi(3.77)$ can be found using the existing experimental data about the radiative decays: $J / \psi \rightarrow \eta_{c} \gamma, \psi(3.77) \rightarrow \eta_{c} \gamma, \eta_{c} \rightarrow \rho \rho, \rho \rightarrow e^{+} e^{-}, J / \psi \rightarrow e^{+} e^{-}$and $\psi(3.77) \rightarrow$ $e^{+} e^{-}$. The details of the calculation are given in the Appendix. On the other hand, using relations (11) and (12), it is possible to find the constants $g_{V}$ of the $\psi(4.04)$ and the $\psi(4.41)$ resonances. As two choices are possible for the sign of $F(0)$ and for the constants $g_{V}$ for the first three resonances, $\rho, J / \psi$ and $\psi(3.77)$, one finds a $2^{4}=16$-fold parametrisation for $F(t)$, which prevents any realistic estimation of the $t$-behaviour of the cross section for the process $e^{+} e^{-} \rightarrow \eta_{c} \gamma$. So our prediction of the various characteristics of the decay $\eta_{c} \rightarrow \gamma e^{+} e^{-}$will be based on the simplified two-pole model for $F(t)$.

\section{B. QCD-Inspired Model}

Now we consider the process of $c \bar{c}$-quark annihilation into $\gamma \gamma^{*}$, which receives the contribution of the two diagrams illustrated in Fig. 5.

In the limit of $|\vec{p}|=0$ (where $\vec{p}$ is the relative momentum of the $c \bar{c}$-system in the CMS), the $c \bar{c}$ - annihilation in a singlet $S$-state - which has the quantum numbers of the $\eta_{c}$-meson, $J^{P}=0^{-}$- is described by a matrix element of the form

$$
\mathcal{M}(c \bar{c}) \propto \vec{k} \cdot \vec{e} \times \overrightarrow{e^{\prime}}
$$

where $\vec{e}\left(\overrightarrow{e^{\prime}}\right)$ is the polarisation vector of the real (virtual) photon. The following relations hold: $\overrightarrow{e^{\prime}} \cdot \vec{k} \neq 0$ and $\vec{e} \cdot \vec{k}=0$. The result (15) can be found, without specific calculations, on the basis of symmetry properties relative to $C$ - and $P$-transformations for the process $c \bar{c} \rightarrow \gamma \gamma^{*}$. Due to the positive $C$-parity of the two final $\gamma^{\prime}$ s, the sum of the total spin $S$ and the angular momentum $\ell$ of the $(c \bar{c})$-system must be even. But in the limit of $\vec{p} \rightarrow 0$ we have $\ell=0$, therefore $S=0$. In this limit the $t$-dependence of the resulting matrix element for $c \bar{c} \rightarrow \gamma \gamma^{*}$ can be identified with the $t$-dependence of the form factor $F(t)$ of the decay $\eta_{c} \rightarrow \gamma \gamma^{*}$. This dependence can be written in terms of the $c$-quark propagators in the elementary process $c \bar{c} \rightarrow \gamma \gamma^{*}$, which are identical for both diagrams of Fig. 5. Therefore in the limit of $\vec{p}=0$ we have

$$
F(t) \propto \frac{1}{(p-k)^{2}-m_{c}^{2}}=\frac{1}{k^{2}-2 p \cdot k} \stackrel{\vec{p}=0}{=} \frac{1}{k^{2}-2 m_{c} k_{0}}=\frac{2}{k^{2}-4 m_{c}^{2}},
$$

which implies that $F(t) \propto|t|^{-1}$ for large $|t|$, unlike the VDM.

The parameter $4 m_{c}^{2}$ which appears in the denominator should be identified with a physical quantity. The simplest possibility is to set $2 m_{c}=m_{J / \psi}$, which corresponds to the $J / \psi$ contribution to $F(t)$, appearing in the framework of VDM. A perturbative approach, on the basis of a factorisation of short- and long-distance physics [20], as considered by Feldmann and Kroll [21,22] in the space-like region, results in

$$
4 m_{c}^{2} \rightarrow m^{2}+2<\vec{k}_{\perp}^{2}>
$$


where $\vec{k}_{\perp}$ denotes the transverse momentum of the $c$-quark in the $\eta_{c}$-meson 23]. This substitution may be regarded as a possible procedure for taking into account the transverse momentum of the quark inside the meson. On the other hand, note that our QCD-inspired consideration above is valid for any $t$, both space-like and time-like, except for a small neighbourhood of $t=4 m_{c}^{2}$. Therefore, if we assume that the substitution (16) can be extended at least up to $t \leq m^{2}, F(t)$ turns out to be very sensitive to $\left\langle\vec{k}_{\perp}^{2}\right\rangle$ near the upper kinematical limit of the Dalitz plot, as results from Fig. 6.

In this approach it is also possible to find the corresponding form factors of the radiative decays $\chi_{J} \rightarrow \gamma \ell^{+} \ell^{-}$of the P-wave charmonium states, $J=0,1$ and 2 . To this end it is necessary to find the matrix element of the process $c \bar{c} \rightarrow \gamma \gamma^{*}$ (see Fig. 5). The spin structure of the $\chi$-decay matrix elements is

$$
\begin{gathered}
\xi_{1}^{\dagger} \sigma_{2} \vec{\sigma} \cdot \vec{p} \xi_{2} \vec{e} \cdot \overrightarrow{e^{\prime}}: \quad \chi_{0} \rightarrow \gamma+\gamma^{*}, \\
\xi_{1}^{\dagger} \sigma_{2} \vec{\sigma} \times \vec{p} \cdot \vec{e} \times \vec{k} \xi_{2} \overrightarrow{e^{\prime}} \cdot \vec{k}: \quad \chi_{1} \rightarrow \gamma+\gamma^{*}, \\
\xi_{1}^{\dagger} \sigma_{2}\left(\sigma_{a} p_{b}+\sigma_{b} p_{a}-\frac{2}{3} \delta_{a b} \vec{\sigma} \cdot \vec{p}\right) \xi_{2}\left\{\begin{array}{cc}
e_{1 a} & e_{1 b} \\
k_{a} k_{b} & \vec{e} \cdot \overrightarrow{e^{\prime}} \\
e_{1 a} k_{b} & \overrightarrow{e^{\prime}} \cdot \vec{k}
\end{array}\right\}: \quad \chi_{2} \rightarrow \gamma+\gamma^{*},
\end{gathered}
$$

where $\xi_{1(2)}$ is the two-component spinor of the $\bar{c}(c)$ quark.

In the general case the decays of $\chi_{0}$ and $\chi_{1}$ are characterised by one form factor, whereas in the case of $\chi_{2} \rightarrow \gamma \gamma^{*}$ three independent form factors are present. Notice also that the matrix element for $\chi_{1} \rightarrow \gamma \gamma^{*}$ is proportional to $\overrightarrow{e^{\prime}} \cdot \vec{k}$, which vanishes for real photons, in agreement with the Landau-Yang theorem.

As one can see from Fig. 6, the VDM and QCD-inspired model predict essentially different $t$-dependences for the transition form factor $F(t)$ in the whole region $4 m_{\ell}^{2} \leq t \leq$ $m^{2}$, where the decay $\eta_{c} \rightarrow \gamma \ell^{+} \ell^{-}$occurs. The coefficients of internal conversion of the decays $\eta_{c} \rightarrow \gamma e^{+} e^{-}$and $\eta_{c} \rightarrow \gamma \mu^{+} \mu^{-}$are also strongly model-dependent (see Table 1). These properties of $F(t)$ depend essentially on two factors: the role of the $\rho$-meson in the VDM approach and the small mass difference between $\eta_{c}$ and $J / \psi$.

Also in the space-like region the two models describe the form factor quite differently; in particular the QCD-inspired model predicts for $F(t)$ a much slower decrease at increasing $|t|$, in agreement with present data [10].

\section{THE REACTION $e^{+} e^{-} \rightarrow \eta_{c} \gamma$}

The differential cross section in the overall CMS reads

$$
\frac{d \sigma}{d \Omega}=\frac{\overline{|\mathcal{M}|^{2}}}{64 \pi^{2} t} \frac{|\vec{q}|}{|\vec{k}|}=\frac{\overline{|\mathcal{M}|^{2}}}{64 \pi^{2} t}\left(1-\frac{m^{2}}{t}\right), \quad m_{e}=0
$$

where $\vec{k}$ and $\vec{q}$ are the three-momenta of the colliding leptons and of the produced $\gamma$ and $t=\left(k_{1}+k_{2}\right)^{2}$ is the square of the total energy of the colliding particles.

Taking into account Eq. (8), we get 


$$
\frac{d \sigma}{d \Omega}=\frac{\alpha^{2}}{32 m^{2}}\left(1+\cos ^{2} \theta\right)\left(1-\frac{m^{2}}{t}\right)^{3}|F(t)|^{2}, \quad t \geq m^{2}
$$

The cross section for collinear kinematics (i.e. $\theta=0$ or $\pi$ ) is non-vanishing, in agreement with helicity conservation. Indeed, in the limit of $m_{e}=0$, owing to the properties of the electromagnetic current $\bar{u} \gamma_{\mu} u$, we have $\lambda_{i}=\lambda_{e^{-}}+\lambda_{e^{+}}= \pm 1$; on the other hand for a real $\gamma$ we have $\lambda_{f}=\lambda_{\gamma}= \pm 1$.

In any process of the type $e^{+} e^{-} \rightarrow \gamma^{*} \rightarrow h_{1} \overline{h_{2}}$, where $h_{1}$ and $h_{2}$ are hadrons or hadronic states, the one-photon mechanism is characterised by a definite angular dependence:

$$
\frac{d \sigma}{d \Omega}=A(t)+B(t) \cos ^{2} \theta
$$

where $\theta$ is the production angle of $h_{1}$ in the overall CMS and $A(t)$ and $B(t)$ are two structure functions which are quadratic combinations of the electromagnetic form factors of the transition $\gamma^{*} \rightarrow h_{1} \overline{h_{2}}$. In the case considered we have only one form factor for the vertex $\gamma^{*} \rightarrow \eta_{c} \gamma$, therefore $B(t) / A(t)=1$, as results from the angular dependence (18).

The threshold behaviour of the cross section is proportional to $\left(1-\frac{m^{2}}{t}\right)^{3}$ and results from the magnetic dipole radiation in the transition $\gamma^{*} \rightarrow \eta_{c} \gamma$.

The factorisation of the $t$ - and $\cos \theta$-dependences in the differential cross section of $e^{+} e^{-} \rightarrow \eta_{c} \gamma$, Eq. (18), is due to the specific structure of the matrix element and to the choice of the CMS as a reference frame. Therefore the $t$-dependence is the same for any production angle and coincides with the $t$-dependence of the total cross section:

$$
\sigma=\frac{\pi}{6 m^{2}} \alpha^{2}\left(1-\frac{m^{2}}{t}\right)^{3}|F(t)|^{2}
$$

Formulae (18) and (19) can be re-written as

$$
\begin{gathered}
\frac{d \sigma}{d \Omega}=\frac{\alpha}{2 m^{3}} \Gamma_{0}\left(1-\frac{m^{2}}{t}\right)^{3}\left|\frac{F(t)}{F(0)}\right|^{2}\left(1+\cos ^{2} \theta\right)=\frac{\left(1+\cos ^{2} \theta\right)}{16 \pi} 3 \sigma \\
\sigma=\frac{4}{3} \frac{\alpha \pi}{m^{3}} \Gamma_{0}\left(1-\frac{m^{2}}{t}\right)^{3}\left|\frac{F(t)}{F(0)}\right|^{2} .
\end{gathered}
$$

In order to predict the $t$-dependence of the total and differential cross sections, it is necessary to know the form factor $F(t)$ in the region $t \geq m^{2}$, and especially where the contribution of all the $\psi$-resonances is important. From our previous discussion it turns out that the VDM can be applied here.

\section{THE PROCESS $\gamma e^{-} \rightarrow \eta_{c} e^{-}$.}

The direct study of the reaction $\gamma e^{-} \rightarrow \eta_{c} e^{-}$is experimentally difficult due to the large threshold, $E_{\gamma, t h r} \simeq m^{2} / 2 m_{e} \simeq 9 \mathrm{TeV}$. Therefore this process can be studied through the $2 \gamma$ mechanism in the reaction $e^{ \pm} e^{-} \rightarrow e^{ \pm} e^{-} \eta_{c}$ (see Figs. 7 and 1) with a quasi-real photon produced in the $e^{ \pm} \rightarrow e^{ \pm} \gamma$-vertex. In this case, for the process $\gamma e^{-} \rightarrow \eta_{c} e^{-}$, it is necessary 
to consider a particular reference frame with colliding $\gamma$ and $e^{-}$. Let us re-write the cross section of $\gamma e^{-} \rightarrow \eta_{c} e^{-}$in terms of the Mandelstam variables $s, t$ and $u$, which can be defined in the standard form, according to the following notation of the particles four-momenta:

$$
\begin{aligned}
\gamma(q)+e^{-}\left(k_{1}\right) \rightarrow e^{-}\left(k_{2}\right)+\eta_{c}(p), & \\
s & =\left(k_{1}+q\right)^{2}, \\
u & =\left(k_{2}-q\right)^{2}, \\
t & =\left(k_{1}-k_{2}\right)^{2},
\end{aligned}
$$

where $q, p$ and $k_{1}\left(k_{2}\right)$ are the four-momenta of the photon, of $\eta_{c}$ and of the initial (final) electron. In the overall CMS the differential cross section can be written as

$$
\frac{d \sigma}{d \Omega_{\eta}}\left(\gamma e^{-} \rightarrow \eta_{c} e^{-}\right)=\frac{\overline{|\mathcal{M}|^{2}}}{64 \pi^{2} s}\left(1-\frac{m^{2}}{s}\right) \text {. }
$$

In the one-photon exchange approximation the expression of $|\overline{\mathcal{M}}|^{2}$ can be derived from eq (5), by exploiting the crossing symmetry. In the direct channel we have

$$
\overline{|\mathcal{M}|^{2}}=\frac{e^{4}|F(t)|^{2}}{m^{2} t}\left(1+\cos ^{2} \theta\right)\left(t-m^{2}\right)^{2},
$$

where $t$ is the overall CMS energy squared.

Taking into account the relation

$$
1-\cos ^{2} \theta=\frac{4 s u}{\left(t-m^{2}\right)^{2}}
$$

the invariant differential cross section for $\gamma e^{-} \rightarrow e^{-} \eta_{c}$ results to be

$$
\frac{d \sigma}{d t}=\frac{\pi \alpha^{2}}{4 s^{2} m^{2}|t|}\left[\left(t-m^{2}\right)^{2}-2 u s\right]|F(t)|^{2}=4 \pi \frac{\alpha}{m^{3}} \Gamma_{0}\left|\frac{F(t)}{F(0)}\right|^{2} \frac{\left(t-m^{2}\right)^{2}-2 u s}{s^{2}|t|} .
$$

Since the four-momentum transfer in this process is space-like, the reaction considered is especially sensitive to the $\rho$-meson contribution, which is the lightest vector meson - in particular for $|t| \leq 1 \mathrm{GeV}^{2}$.

In the framework of one-photon mechanism a definite prediction for the polarisation observables can be derived. Independently of the kinematical conditions, the absorption asymmetry of a linearly polarised photon, i. e.,

$$
\Sigma=\frac{\sigma_{\perp}-\sigma_{\|}}{\sigma_{\perp}+\sigma_{\|}}
$$

where $\sigma_{\perp}\left(\sigma_{\|}\right)$is the differential cross section with linearly polarised photons, whose polarisations are orthogonal (parallel) to the reaction plane - is equal to 1 . This result, which implies $\sigma_{\|}=0$, follows from parity conservation.

This prediction can be tested using a linearly polarised photon beam and detecting the final electron. The electron polarisation here does not carry any additional information, owing to the presence of a single electromagnetic form factor. However for two-spin polarisation observables non-trivial effects can be produced by the collisions of circularly polarised photons with longitudinally polarised electrons. 


\section{CONCLUSIONS}

We have considered some characteristics of the radiative decay $\eta_{c} \rightarrow \gamma \ell^{+} \ell^{-}$, such as the Dalitz distribution with respect to the energies of the two final leptons, the effective mass spectrum of the lepton pair and the coefficient of internal conversion, i.e. the ratio $\Gamma\left(\eta_{c} \rightarrow \gamma \ell^{+} \ell^{-}\right) / \Gamma\left(\eta_{c} \rightarrow 2 \gamma\right)$. We have used two different models for the electromagnetic form factor $F(t)$. The VDM allows to find a relatively simple 2-pole representation for the form factor $F(t)$, with a definite normalisation at $t=0$ and asymptotic behaviour in $t^{-2}$, in agreement with the quark counting rule. The VDM parametrisation contains the contribution of the $\rho$-meson (which is important owing to the large width of the decay $\eta_{c} \rightarrow \rho^{0} \rho^{0}$ ) and the $J / \psi$-contribution. This two-pole representation can be used for analysing the decay $\eta_{c} \rightarrow \gamma \ell^{+} \ell^{-}$, which corresponds to timelike $t$, and the photoproduction process $\gamma e^{-} \rightarrow \eta_{c} e^{-}$, where $t$ is space-like. However in the calculation of the energy dependence of the differential and total cross section of the process $e^{+} e^{-} \rightarrow \eta_{c} \gamma$ the form factor $F(t)$ could be sensitive to the whole set of $\psi$-resonances. A suitable parametrisation of the form factor, in terms of the contribution of the vector mesons $\rho, J / \psi, \psi(3.77), \psi(4.04)$ and $\psi(4.41)$, can be found in the framework of VDM, but with a $2^{4}=16$-fold ambiguities in the definition of the signs of the various constants. The QCD-inspired model for the form factor $F(t)$ on the basis of the process $c \bar{c} \rightarrow \gamma \ell^{+} \ell^{-}$(with two pole Feynman diagrams) results in a parametrisation which is very similar to the $J / \psi$-contribution alone. This model predicts an asymptotic behavour of the type $|t|^{-1}$ for large $|t|$, in contrast with the VDM. 


\section{APPENDIX:}

Here we consider the decays $\eta_{c} \rightarrow \rho^{0} \rho^{0}, V \rightarrow \eta_{c} \gamma$ and $V \rightarrow \ell^{+} \ell^{-}$, whose decay constants are necessary for the calculation of the form factor $F(t)$ in VDM.

The matrix element for the process $\eta_{c} \rightarrow \rho^{0} \rho^{0}$ can be written as

$$
\mathcal{M}=\frac{g\left(\eta_{c} \rightarrow \rho^{0} \rho^{0}\right)}{m} \epsilon^{\mu \nu \alpha \beta} U_{1 \mu} U_{2 \nu} p_{1 \alpha} p_{2 \beta}
$$

where $U_{1}\left(U_{2}\right)$ and $p_{1}\left(p_{2}\right)$ are the polarisation four-vectors and four-momenta of the two produced particles. Summing over the final polarisations, the partial decay width reads

$$
\Gamma\left(\eta_{c} \rightarrow \rho^{0} \rho^{0}\right)=\frac{m}{64 \pi}\left|g\left(\eta_{c} \rightarrow \rho^{0} \rho^{0}\right)\right|^{2}\left(1-\frac{4 m_{\rho}^{2}}{m^{2}}\right)^{3 / 2}
$$

the constant $g\left(\eta_{c} \rightarrow \rho^{0} \rho^{0}\right)$ being dimensionless in the present normalisation.

The matrix element of the decay $V \rightarrow \ell^{+} \ell^{-}$(see Fig 8, which illustrates the notation of the particle four-momenta), can be written as

$$
\mathcal{M}=e^{2} f_{V} \bar{u}\left(k_{1}\right) \gamma_{\mu} u\left(k_{2}\right) U^{\mu}
$$

where $U_{\mu}$ is the polarisation four-vector of the $V$-meson. Averaging over $U_{\mu}$ and summing over the final lepton polarisations, we find, in the limit of zero leptonic mass,

$$
\Gamma\left(V \rightarrow \ell^{+} \ell^{-}\right)=\alpha^{2} f_{V}^{2} \frac{4 \pi}{3} m_{V}
$$

The matrix element of the decay $V \rightarrow P \gamma$, where $P$ is a pseudoscalar meson (Fig 9), can be written as

$$
\mathcal{M}=e \frac{g(V \rightarrow P \gamma)}{m_{V}} \epsilon^{\mu \nu \alpha \beta} U_{\mu} e_{\nu} q_{\alpha} k_{\beta}
$$

Summing over the $\gamma$-polarisations and averaging over the $V$-polarisations yields

$$
\Gamma(V \rightarrow P \gamma)=\frac{\alpha g^{2}(V \rightarrow P \gamma)}{24} m_{V}\left(1-\frac{m^{2}}{m_{V}^{2}}\right)^{3} .
$$

Finally $g_{V}$ is connected to $g(V \rightarrow P \gamma)$ and $f_{V}$ through the relation

$$
g_{V}=e f_{V} g(V \rightarrow P \gamma) m_{V}^{2}
$$

We stress that this relation does not fix the sign of the constant $g_{V}$. 


\section{REFERENCES}

[1] A. Le Yaouanc, Ll. Olivier, O. Pene and J.C. Reynal, Hadron Transitions in the Quark Model, Gordon and Breach Science Publishers, London, 1988; Chapt. 2

[2] T. Appelqvist et al., Ann. Rev. Nucl. Part. Sci. 28, 387 (1978)

[3] H. Fonvieille et al., Phys. Lett. B233, 65 (1989)

[4] CLEO collaboration, V. Savinov et al., (1995) hep-ex/9507005

[5] CLEO collaboration, J. Gronberg et al., Phys. Rev. D57, 33 (1998)

[6] R. Jakob, P. Kroll and M. Raulfs, J. Phys. G22, 45 (1996)

[7] P. Kroll and M. Raulfs, Phys. Lett. B387, 848 (1996)

[8] I. V. Musatov and A. V. Radyushkin, hep-ph/9702443 (1997)

[9] J. Botts and G. Sterman, Nucl. Phys. B325, 62 (1989)

[10] L3 collaboration, M. Acciarri et al., Phys. Lett. B461, 155 (1999)

[11] Particle Data Group, C. Caso et al., EPJ, C3, 1 (1998)

[12] V.A. Novikov et al., Phys. Rep. 41, 1 (1978)

[13] L.J. Reinders, H.R. Rubinstein and S. Yazaki, Phys. Rep. 127, 1 (1985)

[14] K.T. Chao, Nucl. Phys. B335, 101 (1990)

[15] L.J. Reinders, H.R. Rubinstein and S. Yazaki, Phys. Lett. B113, 414 (1982)

[16] E. Di Salvo and M. Pallavicini, Nucl. Phys. B427, 22 (1994)

[17] T. M. Aliev, E. Iltan, N. K. Pak, M. P. Rekalo, Z. Phys. C54, 683 (1994)

[18] A. Z. Dubnickova, S. Dubnicka and M. P. Rekalo, Z. Phys. C70, 473 (1996)

[19] N. M. Kroll, W. Wada, Phys. Rev. 98, 1355 (1955)

[20] G. P. Lepage and S. J. Brodsky, Phys. Rev. D22, 2157 (1980)

[21] T. Feldmann and P. Kroll, Phys. Lett. B413, 410 (1997)

[22] T. Feldmann, "Fourth International Workshop on Progress in Heavy Quark Physics", Sept. 20-22, 1997 (hep-ph/9710385); T. Feldmann and P. Kroll, "PHOTON '97" Egmond aan Zee, The Netherlands, May 10-15, 1997 (hep-ph/9706224)

[23] M. Wirbel, B. Steeh and M. Bauer Z. Phys. C29, 637 (1985) 


\section{TABLES}

\begin{tabular}{|c|c|c|}
\hline \hline Model & $\eta_{c} \rightarrow \gamma e^{+} e^{-}$ & $\eta_{c} \rightarrow \gamma \mu^{+} \mu^{-}$ \\
\hline \hline VDM & 3.99 & 2.39 \\
\hline \hline QCD,$\Delta=.075$ & 2.22 & 0.61 \\
QCD,$\Delta=.100$ & 2.22 & 0.60 \\
QCD,$\Delta=.125$ & 2.21 & 0.69 \\
\hline \hline
\end{tabular}

TABLE I. Prediction for the coefficients of internal conversion in units of $10^{-2}$. We have set $\Delta=2<\vec{k}_{\perp}^{2} / m^{2}>$. 


\section{FIGURES}

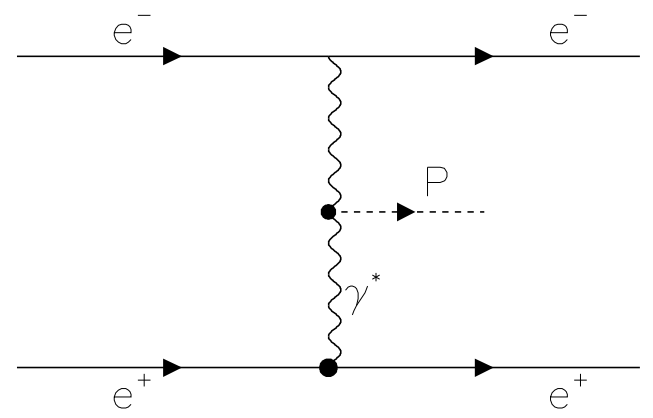

FIG. 1. $2 \gamma$-mechanism for the $e^{+} e^{-} \rightarrow e^{+} e^{-} P$-process.

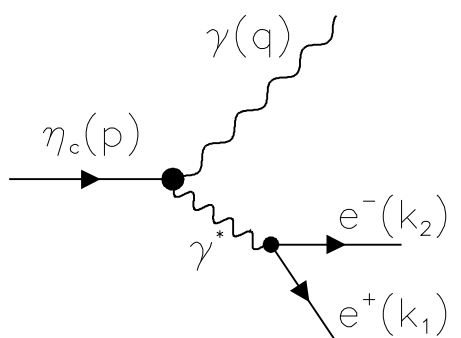

FIG. 2. Diagram for the process $\eta_{c} \rightarrow \gamma e^{+} e^{-}$, with notation of particles four-momenta.

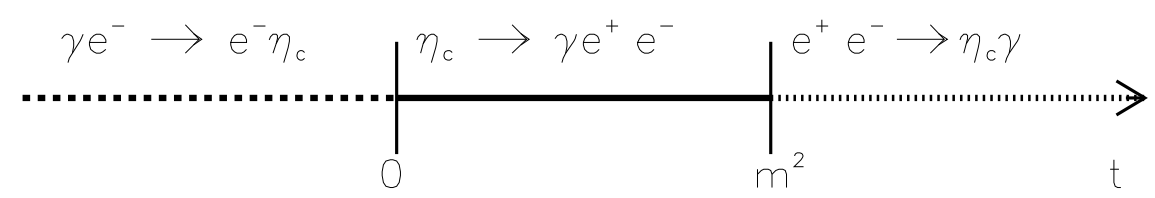

FIG. 3. Scheme for the momentum transfer regions covered by the reactions $\gamma e^{-} \rightarrow \eta_{c} e^{-}$, $\eta_{c} \rightarrow \gamma e^{+} e^{-}$and $e^{+} e^{-} \rightarrow \eta_{c} \gamma$. 


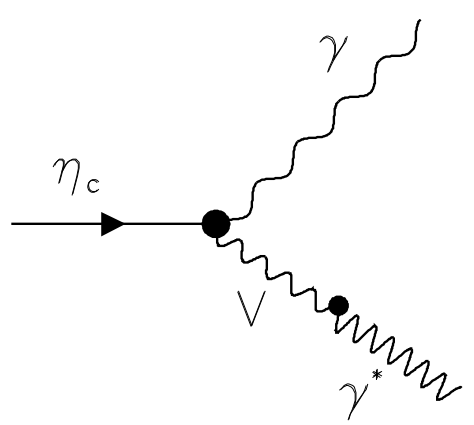

FIG. 4. Diagram for the process $\eta_{c} \rightarrow \gamma \gamma^{*}$ in framework of VDM.
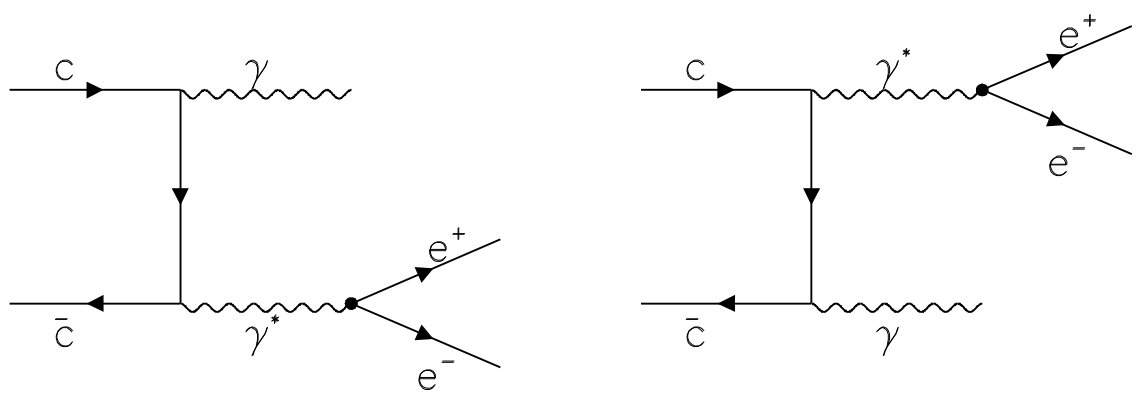

FIG. 5. Diagrams for the process $c \bar{c} \rightarrow \gamma e^{+} e^{-}$. 


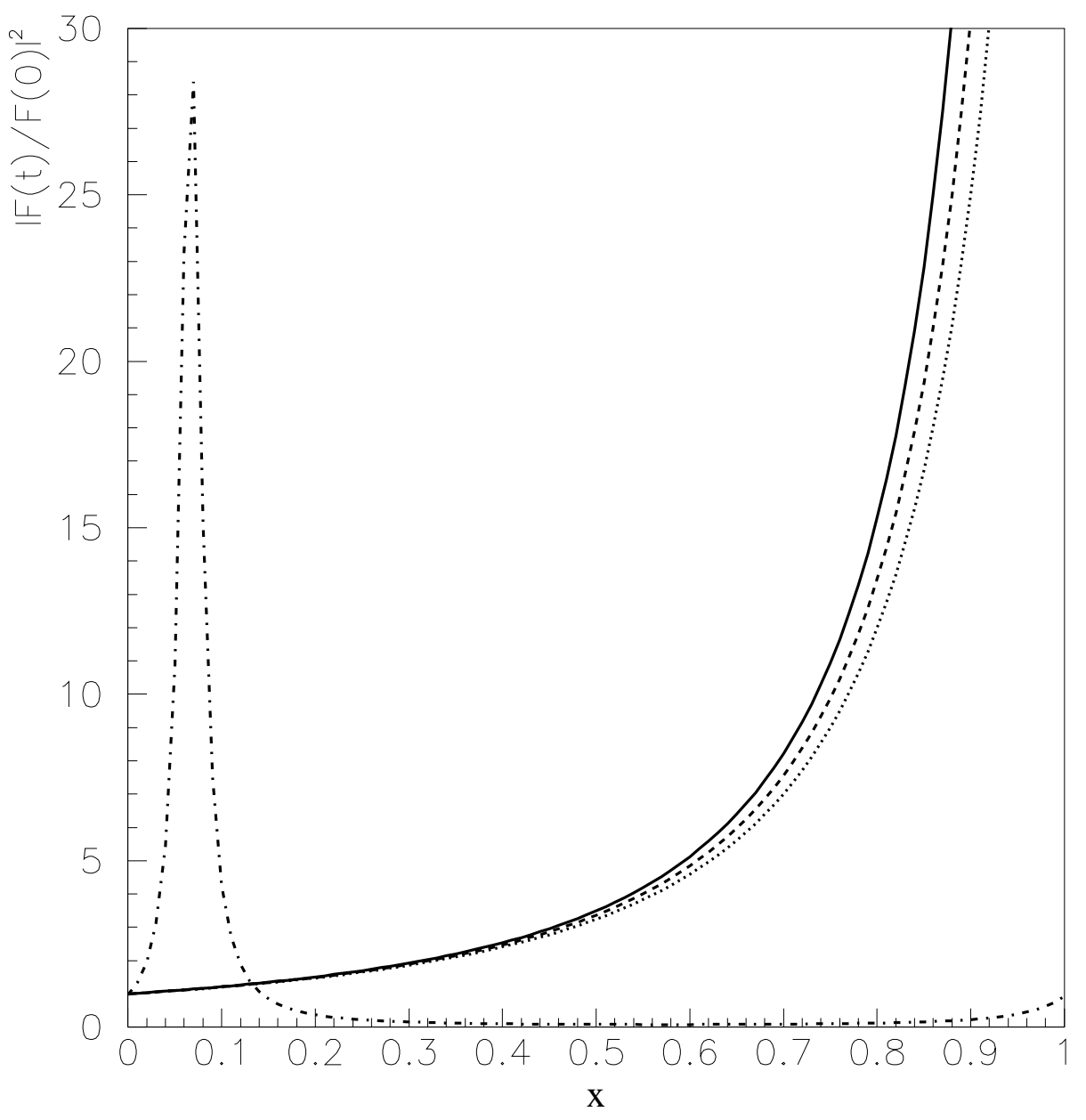

FIG. 6. $\mathbf{x}$-dependence $\left(\mathbf{x}=t / m^{2}\right)$ of $|F(t) / F(0)|^{2}$ in the VDM two-pole model (dashed-dotted line) and in the QCD-inspired model for different values of the transverse quark momentum: $2<\vec{k}_{\perp}^{2}>/ m^{2}=0.075$ (solid line), $2<\vec{k}_{\perp}^{2}>/ m^{2}=0.100$ (dashed line), and $2<\vec{k}_{\perp}^{2}>/ m^{2}=0.125$ (dotted line). 


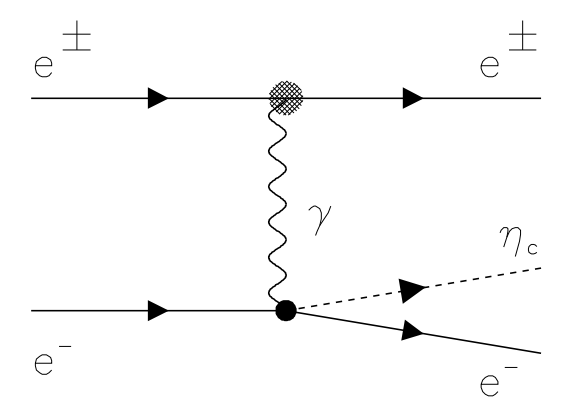

FIG. 7. Feynman diagram for $e^{ \pm} e^{-} \rightarrow e^{ \pm} e^{-} \eta_{c}$. 


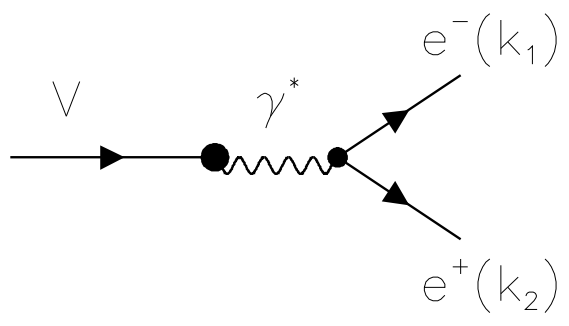

FIG. 8. Diagram of the decay $V \rightarrow e^{+} e^{-}$, with notation of particles four-momenta, in the framework of one-photon exchange mechanism. 


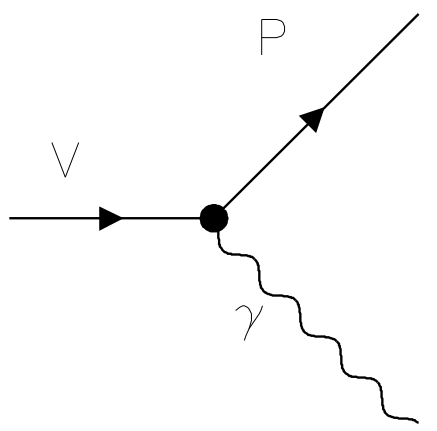

FIG. 9. Diagram of the decay $V \rightarrow P \gamma$. 